\title{
Differential presentations of arterial thromboembolic events between venous thromboembolism and atrial fibrillation patients
}

\author{
Yu-Sheng Lin ${ }^{1}$, Ming-Shyan Lin ${ }^{2}$, Chien-Chia $\mathrm{Wu}^{3}$, Yung-Lung Chen ${ }^{4}$, Jung-Jung Chang ${ }^{1}$, \\ Pao-Hsien $\mathrm{Chu}^{3}$, Gregory Lip ${ }^{5}$, and Mien-Cheng $\mathrm{Chen}^{4}$ \\ ${ }^{1}$ Chiayi Chang Gung Memorial Hospital \\ ${ }^{2}$ Chang Gung Memorial Hospital Chiayi Branch \\ ${ }^{3}$ Chang Gung Memorial Hospital \\ ${ }^{4}$ Chang Gung Memorial Hospital Kaohsiung Branch \\ ${ }^{5}$ University of Liverpool
}

February 19, 2021

\begin{abstract}
Background: Atrial fibrillation (AF) and venous thromboembolism (VTE) share several risk factors related to arterial thromboembolism. No study has reported the differential contribution to arterial thromboembolic events and mortality between these two conditions in the same population. Methods: We included AF and VTE national cohorts derived from Taiwan National Health Insurance Research Database between 2001 and 2013. The eligible population was 314,861 patients in the AF cohort and 41,102 patients in the VTE cohort. The primary outcome was arterial thromboembolic events, including ischemic stroke, extracranial arterial thromboembolism (ECATE) and myocardial infarction (MI). Secondary outcomes were all-cause mortality and cardiovascular death. Results: After a 1:1 propensity matching, 32,688 patients in either group were analyzed. The risk of arterial thromboembolic events was lower in the VTE cohort than that in the AF cohort (subdistribution hazard ratio [SHR], $0.60 ; 95 \%$ confidence interval [CI], 0.57-0.62)). The risk of ischemic stroke (SHR, 0.44; 95\% CI, 0.42-0.46) and MI (SHR, 0.80; 95\% CI, 0.72-0.89) were lower in the VTE cohort, while the risk of ECATE (SHR, 1.23; 95\% CI, 1.14-1.33; particularly lower extremities) was higher in the VTE cohort. All-cause mortality rate was higher in the VTE cohort (HR, 1.18; 95\% CI, 1.15-1.21) while the risk of cardiovascular death was lower in the VTE cohort (HR, 0.96; 95\% CI, 0.93-0.995). Conclusions: Patients with AF had higher risks of arterial thromboembolic events compared to patients with VTE, despite having risk factors in common. The VTE cohort had higher risks of all-cause mortality and ECATE, particularly lower extremity events, compared to AF patients. These differential manifestations of thromboembolism sequelae in AF and VTE merit further investigation.
\end{abstract}

\section{Introduction}

Atrial fibrillation (AF) is associated with an increased risk of stroke, systemic thromboembolic events, and mortality. ${ }^{1}$ Long-term anticoagulation therapy, particularly with direct oral anticoagulants (DOACs), significantly reduces the risk of stroke and mortality. ${ }^{2}$ In terms of venous thromboembolism (VTE), including deep venous thrombosis (DVT) and pulmonary embolism(PE), the duration of anticoagulation therapy to prevent recurrences takes into consideration the risk of recurrent VTE and the risk of bleeding. ${ }^{3}$

AF and VTE have many pathophysiological and clinical risk factors in common. For example, the pathogenesis of thromboembolism in AF has been associated with a prothrombotic state by fulfilling Virchow's triad for thrombogenesis, with abnormal blood flow (stasis), vessel wall abnormalities (structural heart disease) and abnormal blood constituents (coagulation factors). ${ }^{4}$ The pathogenesis of VTE also has similar predisposing factors. ${ }^{3,5}$ Patients with AF and/or VTE have many cardiovascular risk factors, such as hypertension, smoking, hyperlipidemia, diabetes, and obesity ${ }^{6-8}$ and malignancy. ${ }^{9}$ VTE has also been reported to be associated 
with several cardiovascular diseases, including peripheral artery disease and myocardial infarction (MI) ${ }^{10,11}$ as well as non-cardiovascular factors, such as malignancy. ${ }^{12,13}$ Indeed, one community registry study reported that AF and VTE independently contributed to each other. ${ }^{14}$ Thus, venous and arterial thrombosis may be different sides of the same coin. ${ }^{15}$

Of note, the duration of prescribing anticoagulation is quite different between AF and VTE in current practice. Long-term anticoagulation should be prescribed for AF patients ${ }^{2,16}$ whereas more limited-duration of anticoagulation is sometimes prescribed for VTE patients unless there are high risk features for recurrence. ${ }^{3,17}$

We hypothesized that AF and VTE, despite sharing many pathophysiological and clinical risk factors, would have differential contribution to arterial thromboembolic events and mortality in the same population. Accordingly, we tested this hypothesis in a nationwide cohort study of VTE and AF patients from the Taiwan National Health Insurance Database.

\section{Methods}

The data of this national retrospective cohort study was retrieved from the Taiwan National Health Insurance Research Database (NHIRD) released by the Taiwan National Health Research Institutes. The National Health Insurance system is a mandatory universal health insurance program that offers comprehensive medical care coverage to nearly all Taiwan residents since the inception of the program in March 1995. In the NHIRD, the patients' original identification numbers are encrypted and the encrypting procedure is consistent, so that linking claims belonging to the same enrollee is feasible and can be followed longitudinally. The available health care information included complete outpatient visits, hospitalization, and diseases, which were registered using International Classification of Diseases, Ninth Revision, Clinical Modification (ICD-9$\mathrm{CM}$ ) codes. ${ }^{18}$ In addition, medication prescriptions are also recorded. Patients with newly diagnosis of AF and VTE were included in this study. The study was approved by the Institutional Review Board of Chang Gung Memorial Hospital (201900915B1).

\section{Identification of patients with VTE and AF}

This study included national AF and VTE cohorts. Patients with AF were identified with [?]2 times outpatient visits or in a discharge diagnosis using the ICD-9 CM diagnostic code of 427.31 between 2001 and 2013. Patients with VTE were identified using the discharge diagnosis (ICD-9-CM: 453 for DVT and 415.1 for PE) with use of anticoagulation during admission between 2001 and December 31, 2013. In the AF cohort, we excluded patients who were under age of 20 years old and were diagnosed as VTE historically or in follow-up period. In the VTE cohort, we excluded patients who were under age of 20 years old and were diagnosed as $\mathrm{AF}$ in the follow-up period. In order to compare the differences of clinical outcomes after developing $\mathrm{AF}$ and VTE, we excluded those who died at the index admission in both cohorts. Finally, 314,861 AF patients without VTE and 41,102 VTE patients without AF were included in this study (Figure 1).

\section{Covariates}

Covariates were age, sex, eighteen comorbidities, Charlson Comorbidity Index score, four historical events, and fourteen kinds of medications (Table 1). Comorbidities were recognized with at least two clinic visits or anyone inpatient record in the previous year before the index date. Historical events were detected using anyone inpatient diagnosis before the index date which could be tracked up to year 1997. The use of medication was extracted within 3 months after index date. All the information about medications were extracted from the claims data of outpatient visits or the refill for chronic illness in the pharmacy by using the Anatomical Therapeutic Chemical codes or the Taiwan NHI reimbursement code.

\section{Outcomes}

The primary outcome was arterial thromboembolic events, including ischemic stroke, myocardial infarction (MI) and extracranial arterial thromboembolism (ECATE). Extracranial arterial thromboembolism included arterial thromboembolic occlusion of an extremity or extracranial vital organ, including kidney, intestine, and spleen. The occurrence of ischemic stroke and MI was defined as the principal discharge diagnosis of 
hospitalization. The occurrence of ECATE was defined as the principal or secondary diagnoses of hospitalization. Secondary outcomes were all-cause mortality and cardiovascular death. All-cause mortality was defined as withdrawal from the NHI program. ${ }^{19}$ The definition of cardiovascular (CV) death was the criteria of the Standardized Definitions for Cardiovascular and Stroke Endpoint Events in Clinical Trials by the FDA in the United States. Each patient was followed from the discharge date of index admission to the date of event occurrence, date of death, or December 31, 2013.

\section{Ascertainment of VTE, AF, ischemic stroke and ECATE}

The validation of $\mathrm{AF}$ has been assessed and presented in our previous reports, with a high positive predictive value (PPV) of $89 \% .{ }^{20}$ The accuracy of VTE was reliable in the Taiwan insurance claim system and some published studies also used the same diagnosis method. ${ }^{10,21}$ Ischemic stroke was also validated with high PPVs. ${ }^{22}$ In terms of ECATE, a validation study was conducted at our medical center, randomly sampling 100 hospitalizations for systemic thromboembolism who were selected using the same criteria as mentioned in this study. After experienced physicians (YSL and VCCW) reviewed the medical records and all imaging results, including vascular duplex, computed tomography angiography and intervention reports, the PPV of systemic thromboembolism was $88 \%$ (data not shown).

\section{Statistics}

There would be substantial difference in the baseline characteristics between study groups (i.e., VTE and AF cohorts). Therefore, we performed 1:1 ratio propensity score matching to make the covariates balanced between groups. The propensity score was the predicted probability to be in the one group (i.e., VTE) given the values of covariates using the multivariable logistic regression without considering interaction effects. The variables selected to calculate propensity score were listed in Table 1 where the follow-up year was replaced with the index date (Table 1). The matching was processed using a greedy nearest neighbor algorithm with a caliper of 0.2 times of the standard deviation of the logit of propensity score, with random matching order and without replacement. The quality of matching was checked using the absolute value of standardized difference (STD) between the groups, where a value less than 0.1 was considered negligible difference. We additionally performed three propensity score matchings to compare the PE-only cohort with the DVT-only cohort, the DVT-only cohort with the AF cohort and the PE-only cohort with the AF cohort, respectively.

As to the time to fatal outcomes (i.e., all-cause mortality and cardiovascular death), the risks between the groups were compared by the Cox proportional hazard model. The incidences of time to non-fatal outcomes (e.g., ischemic stroke or MI) between groups were compared by the Fine and Gray subdistribution hazard model which considered all-cause mortality a competing risk. The within-pair clustering of outcomes after propensity score matching was accounted for by using a robust standard error. ${ }^{23} \mathrm{~A}$ two-sided $P$ value $<0.05$ was considered statistically significant. Statistical analyses were performed using SAS version 9.4 (SAS Institute, Cary, NC).

\section{Results}

\section{Baseline characteristics}

This study enrolled 314,861 AF patients (mean age of 71.2 \pm 13.5 years) and 41,102 VTE patients (mean age of $64.3 \pm 16.4$ years). These two cohorts were different in age distribution, whereby the VTE cohort was predominantly age $<65$ years while the AF cohort was predominantly age [?] 75 years (Table 1 ). The AF cohort had significantly greater prevalence of hypertension, ischemic heart disease, chronic obstructive pulmonary disease (COPD), hyperthyroidism and heart failure, while VTE cohort had higher prevalence of peripheral artery disease, cancer and auto-immune disease. In terms of medications, angiotensin converting enzyme inhibitors/angiotensin receptor blockers, beta blockers and digoxin were more frequently prescribed in the AF population. In addition, the available follow-up period in AF cohort were longer than that in VTE cohort (AF cohort vs VTE cohort: 4.2 +- 3.4 vs 3.8 +- 3.5 years, standardized difference: -0.12 ).

After matching, 32,688 patients in either cohort were well balanced in baseline characteristics, including follow-up period (Table 1). In patients with DVT-only versus AF, the AF cohorts had higher incidence 
of ischemic heart disease, heart failure and COPD while DVT-only cohort had higher incidence of cancer, auto-immune disease and peripheral artery disease. (Supplemental table 1) In patients with PE-only versus AF, the PE-only cohort had a higher incidence of cancer. ( Supplemental table 2) In terms of the PE-only versus DVT-only cohorts, the PE-only cohort had higher incidence of ischemic heart disease, heart failure and COPD while DVT-only cohort had higher prevalence of cancer and peripheral artery disease.( Supplemental table 3 )

\section{Outcomes between VTE and AF cohorts}

The outcomes between VTE and AF cohort obtained After propensity matching are shown in Supplemental table 4 and Figure 2. The risk of the arterial thromboembolic events was lower in the VTE cohort (subdistribution hazard ratio [SHR], 0.60; 95\% confidence interval [CI], 0.57-0.62) (Figure 2A), as were the risks of ischemic stroke (SHR, 0.44; 95\% CI, 0.42-0.46) (Figure 2B) and MI (SHR, 0.80; 95\% CI, 0.72-0.89). The risk of ECATE (SHR, 1.23; 95\% CI, 1.14-1.33) (Figure 2C) and all-cause mortality rate (HR, 1.18; 95\% CI, 1.15-1.21) was higher in VTE cohort (Figure 2E), although the latter had lower CV death (HR, 0.96; $95 \%$ CI, 0.93-0.995) (Figure 2D).

In subgroup analysis of the cause of death after propensity matching, the percentage of CV death was significant higher in AF cohort than in VTE cohort (VTE cohort versus AF cohort: $56.4 \%$ versus $66.6 \%$, $\mathrm{P}<0.001$ ). (Supplemental table 5) The percentages of death related to cancer and infection other than pneumonia among the causes of non-CV death were significantly higher in the VTE cohort than in the AF cohort.

\section{Outcomes between DVT-only and AF cohorts}

After propensity matching, there was no substantial difference in the baseline characteristics between the DVT-only and AF cohorts (Supplemental table 1). Clinical outcomes between DVT-only and AF cohorts are shown in Supplemental table 6.

The incidence of arterial thromboembolic event was lower in the DVT-only cohort than that in the AF cohort (SHR, 0.62; 95\% CI, 0.59-0.65) (Figure 3A). The risks of ischemic stroke (SHR, 0.45; 95\% CI, 0.43-0.48) (Figure 3B) and MI (SHR, 0.76; 95\% CI, 0.67-0.86) were lower, while the risk of ECATE was higher, in the DVT-only cohort (SHR, 1.31; 95\% CI, 1.20-1.43) (Figure 3C). All-cause mortality rates were higher in the DVT-only cohort (HR, 1.14; 95\% CI, 1.11-1.17), while the rate of CV death was lower (HR, 0.89; 95\% CI, 0.85-0.92) (Figure 3D and Figure 3E).

\section{Outcomes between PE-only and AF cohorts}

After propensity matching, there was no substantial difference in the baseline characteristics between the $\mathrm{PE}$ alone and AF cohorts (Supplemental table 2). Clinical outcomes between PE-only and AF cohorts were shown in Supplemental table 7. The incidence of the arterial thromboembolic event was lower in the PE-only cohort than that in AF cohort (SHR, 0.52; 95\% CI, 0.48-0.56) (Figure 4A). The risk of ischemic stroke was lower in the PE-only cohort (SHR, 0.41; 95\% CI, 0.37-0.45) (Figure 4B) with no differences in ECATE (Figure 4C) and MI event rates (Supplemental table 7). The PE-only cohort had higher all-cause mortality (HR, 1.26; 95\% CI, 1.20-1.32) and CV death (HR, 1.14; 95\% CI, 1.07-1.22) than the AF cohort (Figure 4D and Figure 4E).

\section{Outcomes between PE-only and DVT-only cohorts}

After propensity matching, there was no substantial difference in the baseline characteristics between the PE-only and DVT-only cohorts (Supplemental table 3). Clinical outcomes between PE-only and DVT-only cohorts were shown in Supplemental table 8. The arterial thromboembolic event was lower in the PE-only cohort than in the DVT-only cohort (SHR, 0.80; 95\% CI, 0.73-0.88) (Figure 5A). The risks of ischemic stroke (SHR, 0.82; 95\% CI, 0.74-0.92) and ECATE, including lower extremity events, (SHR, 0.69; 95\% CI, 0.58-0.82) were lower in the PE-only cohort than in the DVT-only cohort (Figure 5B and Figure 5C). The 
risks of all-cause mortality (HR, 1.08; 95\% CI, 1.03-1.13) and CV death (HR, 1.28; 95\% CI, 1.19-1.37) were higher in the PE-only cohort compared to the DVT-only cohort (Figure 5D and Figure 5E).

\section{Discussion}

This retrospective 10-year nationwide cohort study enrolled two national cohorts shows that the arterial thromboembolic events, ischemic stroke and MI, were higher in matched patients with AF cohort than those with VTE cohort. Second, the VTE cohort had higher incidence of ECATE than AF cohort, particularly lower extremity thromboembolism. Third, the AF cohort had higher incidence of CV death, but lower incidence of all-cause mortality compared to the VTE cohort. (Figure 6 and Supplemental table 9) In subgroup analyses comparing the DVT-only, PE-only and AF cohorts, the AF patients had highest incidence of ischemic stroke among the three cohorts and had similar incidence of MI compared to patients with PEonly. Patients with DVT-only had highest incidence of ECATE among the three cohorts, particularly lower extremity thromboembolic event. In terms of mortality, patients with PE-only had highest incidence of CV death and all-cause mortality. (Supplemental table 9)

A national 20-year observational study demonstrated that patients with VTE had a 1.26-1.31fold increased risk of subsequent arterial thromboembolic events, including MI and stroke. ${ }^{11}$ Schulman et al. also showed that VTE was associated with a 1.28 fold increased risk of MI or stroke over a 10-year follow-up period. ${ }^{24}$ Epidemiological studies and meta-analysis have also recognized that AF is independently associated with a five-fold increased risk of stroke ${ }^{1}, 1.47$ fold increased risk of $\mathrm{MI}^{25}$, and a two-fold increased risk of mortality. ${ }^{26}$

Although VTE and AF contribute to similar arterial thromboembolic events, we are unaware of any study that has compared the different presentations of arterial thromboembolic events between VTE and AF patients, from the same population cohort. Based on our study, AF contributed to more arterial thromboembolic events while VTE contributed to greater all-cause mortality. In terms of the causes of mortality, more AF patients (66.6\%) died from cardiovascular death than VTE patients (56.4\%) while more VTE patients (13.7\%) died from cancer than AF patients (8.6\%) (Supplemental table 5). Our results were generally consistent with other studies in terms of causes of death in VTE and AF patients. ${ }^{27}$

As mentioned above there are many parallels between the epidemiology of risk factors and associated pathogenesis of thrombosis in AF and VTE. ${ }^{4-6,8,28}$ Nonetheless, our study showed some differential distributions of baseline characteristics between VTE and AF cohorts. Of note, the prevalence of peripheral artery disease and cancer was higher in VTE cohort than AF cohort. In contrast, the prevalence of ischemic heart disease and heart failure was higher in AF cohort than VTE cohort. Importantly, even after propensity matching, AF cohort had higher risks of arterial thromboembolic event, ischemic stroke and MI compared to the VTE cohort. VTE cohort had higher all-cause mortality while AF cohort had higher CV mortality. Therefore, VTE and AF patients have different risks in presentations related to arterial thromboembolic events; thus, $\mathrm{AF}$ and VTE should not be considered as a different side of exactly the same coin.

Long-term anticoagulation therapy to prevent arterial thromboembolism is a well-established strategy in AF population ${ }^{2}$ and a net clinical benefit more than 5 years with DOACs is still evident. ${ }^{16}$ On the other hand, long-term anticoagulation was not recommended in VTE population due to uncertain net clinical benefit in the era of vitamin K antagonists (VKA, eg. warfarin). ${ }^{17}$ However, some studies have shown that extended treatment with DOAC for 6 months to 15 months resulted in less recurrent VTE events than no treatment, and had less bleeding events compared to VKA. ${ }^{29-32}$ Of note, extended low-dose aspirin in VTE patients for up to 4 years results in a significant reduction in the rate of major vascular events, with improved net clinical benefit in the ASPIRE study. ${ }^{33}$

Given the different presentations of arterial thromboembolic events between AF and VTE cohorts over a 10-year follow-up period, further studies of extended antithrombotic therapy in preventing arterial thromboembolic events, particularly lower extremity thromboembolic events, in VTE patients are warranted.

\section{Limitations}


Our study has several limitations. First, we could not clearly identify the prevalence of provoked and unprovoked VTE in our VTE cohort. Several observational studies have reported that unprovoked VTE does not contribute to the same risk as provoked VTE in terms of clinical outcomes, including arterial thromboembolic events. However, a 20-year national observational cohort study reported no significant differences in arterial CV events between provoked and unprovoked VTE. ${ }^{11}$ In addition, the distinction between provoked/unprovoked PE is no longer supported by the 2019 ESC guidelines for the diagnosis and management of acute pulmonary embolism. ${ }^{34}$ Furthermore, clinical presentations/manifestations and laboratory data were not available in NHIRD and such information might affect the outcomes of VTE, especially those with PE. ${ }^{34}$ In order to reduce the bias, we excluded those died during hospitalization and within 3 months after discharge. Second, differentiating subtypes of AF (paroxysmal, sustained) cannot be performed because this information was not available in our national database. Although the incidence of ischemic stroke is considered to be generally lower in paroxysmal AF patients than in patients with sustained $\mathrm{AF},{ }^{35}$ it should not affect the outcomes between AF and VTE in such a large volume study. Third, the duration of anticoagulation therapy was different between AF versus VTE cohorts. ${ }^{2,34}$ We also did not compare the outcomes between AF and VTE cohorts in individual different scenarios with different durations of anticoagulation therapy.

\section{Conclusion}

Patients with AF had higher risks of arterial thromboembolic events (ischemic stroke and MI) compared to patients with VTE, despite having risk factors in common. The VTE cohort had higher risks of all-cause mortality and ECATE, particularly lower extremity events, compared to AF patients. These differential manifestations of thromboembolism sequelae in AF and VTE merit further investigation.

\section{Acknowledgments}

We would like to thank Alfred Hsing-Fen Lin and Ben Yu-Lin Chou for the statistical assistance during the completion of this manuscript.

\section{Sources of Funding}

None.

\section{Disclosures}

GYHL: Consultant for Bayer/Janssen, BMS/Pfizer, Medtronic, Boehringer Ingelheim, Novartis, Verseon and Daiichi-Sankyo. Speaker for Bayer, BMS/Pfizer, Medtronic, Boehringer Ingelheim, and Daiichi-Sankyo. No fees are directly received personally.

\section{References}

1. Wolf PA, Abbott RD, Kannel WB. Atrial fibrillation as an independent risk factor for stroke: the Framingham Study. Stroke.1991;22(8):983-988.

2. January CT, Wann LS, Calkins H, et al. 2019 AHA/ACC/HRS Focused Update of the 2014 AHA/ACC/HRS Guideline for the Management of Patients With Atrial Fibrillation: A Report of the American College of Cardiology/American Heart Association Task Force on Clinical Practice Guidelines and the Heart Rhythm Society. J Am Coll Cardiol.2019;74(1):104-132.

3. Witt DM, Nieuwlaat R, Clark NP, et al. American Society of Hematology 2018 guidelines for management of venous thromboembolism: optimal management of anticoagulation therapy. Blood advances.2018;2(22):3257-3291.

4. Khan AA, Lip GYH. The prothrombotic state in atrial fibrillation: pathophysiological and management implications. Cardiovascular research. 2019;115(1):31-45.

5. Riva N, Donadini MP, Ageno W. Epidemiology and pathophysiology of venous thromboembolism: similarities with atherothrombosis and the role of inflammation. Thrombosis and haemostasis.2015;113(6):1176- 
1183.

6. Ageno W, Becattini C, Brighton T, Selby R, Kamphuisen PW. Cardiovascular risk factors and venous thromboembolism: a meta-analysis. Circulation. 2008;117(1):93-102.

7. Gregson J, Kaptoge S, Bolton T, et al. Cardiovascular Risk Factors Associated With Venous Thromboembolism. JAMA cardiology.2019;4(2):163-173.

8. Anumonwo JM, Kalifa J. Risk Factors and Genetics of Atrial Fibrillation. Heart failure clinics. 2016;12(2):157-166.

9. Farmakis D, Parissis J, Filippatos G. Insights into onco-cardiology: atrial fibrillation in cancer. $J$ Am Coll Cardiol.2014;63(10):945-953.

10. Chang SL, Huang YL, Lee MC, et al. Association of Varicose Veins With Incident Venous Thromboembolism and Peripheral Artery Disease.JAMA. 2018;319(8):807-817.

11. Sorensen HT, Horvath-Puho E, Pedersen L, Baron JA, Prandoni P. Venous thromboembolism and subsequent hospitalisation due to acute arterial cardiovascular events: a 20-year cohort study. Lancet.2007;370(9601):1773-1779.

12. Schulman S, Lindmarker P. Incidence of cancer after prophylaxis with warfarin against recurrent venous thromboembolism. Duration of Anticoagulation Trial. N Engl J Med. 2000;342(26):1953-1958.

13. Heit JA, O'Fallon WM, Petterson TM, et al. Relative impact of risk factors for deep vein thrombosis and pulmonary embolism: a population-based study. Archives of internal medicine.2002;162(11):1245-1248.

14. Lutsey PL, Norby FL, Alonso A, et al. Atrial fibrillation and venous thromboembolism: evidence of bidirectionality in the Atherosclerosis Risk in Communities Study. Journal of thrombosis and haemostasis.2018;16(4):670-679.

15. Franchini M, Mannucci PM. Venous and arterial thrombosis: different sides of the same coin? European journal of internal medicine.2008;19(7):476-481.

16. Connolly SJ, Wallentin L, Ezekowitz MD, et al. The Long-Term Multicenter Observational Study of Dabigatran Treatment in Patients With Atrial Fibrillation (RELY-ABLE) Study. Circulation.2013;128(3):237-243.

17. Robertson L, Yeoh SE, Ramli A. Secondary prevention of recurrent venous thromboembolism after initial oral anticoagulation therapy in patients with unprovoked venous thromboembolism. The Cochrane database of systematic reviews. 2017;12:Cd011088.

18. Lin LY, Warren-Gash C, Smeeth L, Chen PC. Data resource profile: the National Health Insurance Research Database (NHIRD). Epidemiology and health. 2018;40:e2018062.

19. Wu CY, Chen YJ, Ho HJ, et al. Association between nucleoside analogues and risk of hepatitis B virus-related hepatocellular carcinoma recurrence following liver resection. JAMA.2012;308(18):1906-1914.

20. Chang $\mathrm{CH}$, Lee YC, Tsai CT, et al. Continuation of statin therapy and a decreased risk of atrial fibrillation/flutter in patients with and without chronic kidney disease. Atherosclerosis.2014;232(1):224-230.

21. Wang CC, Lin CL, Wang GJ, Chang CT, Sung FC, Kao CH. Atrial fibrillation associated with increased risk of venous thromboembolism. A population-based cohort study. Thrombosis and haemostasis.2015;113(1):185-192.

22. Hsieh CY, Chen CH, Li CY, Lai ML. Validating the diagnosis of acute ischemic stroke in a National Health Insurance claims database. Journal of the Formosan Medical Association. 2015;114(3):254-259.

23. Austin PC, Fine JP. Propensity-score matching with competing risks in survival analysis. Stat Med. 2019;38(5):751-777. 
24. Schulman S, Lindmarker P, Holmstrom M, et al. Post-thrombotic syndrome, recurrence, and death 10 years after the first episode of venous thromboembolism treated with warfarin for 6 weeks or 6 months. Journal of thrombosis and haemostasis : JTH. 2006;4(4):734-742.

25. Guo XY, Li N, Du X, et al. Atrial fibrillation is associated with an increased risk of myocardial infarction: Insights from a meta-analysis. Atherosclerosis. 2016;254:1-7.

26. Benjamin EJ, Wolf PA, D'Agostino RB, Silbershatz H, Kannel WB, Levy D. Impact of atrial fibrillation on the risk of death: the Framingham Heart Study. Circulation. 1998;98(10):946-952.

27. Novo G, Lee E, Choi E-K, et al. Mortality and causes of death in patients with atrial fibrillation: A nationwide population-based study.PloS one. 2018;13(12).

28. Heit JA. Epidemiology of venous thromboembolism. Nature reviews Cardiology. 2015;12(8):464-474.

29. Schulman S, Kearon C, Kakkar AK, et al. Extended use of dabigatran, warfarin, or placebo in venous thromboembolism. N Engl J Med.2013;368(8):709-718.

30. Agnelli G, Buller HR, Cohen A, et al. Apixaban for extended treatment of venous thromboembolism. N Engl J Med.2013;368(8):699-708.

31. Weitz JI, Lensing AWA, Prins MH, et al. Rivaroxaban or Aspirin for Extended Treatment of Venous Thromboembolism. N Engl J Med.2017;376(13):1211-1222.

32. Raskob G, Ageno W, Cohen AT, et al. Extended duration of anticoagulation with edoxaban in patients with venous thromboembolism: a post-hoc analysis of the Hokusai-VTE study. The Lancet Haematology. 2016;3(5):e228-236 .

33. Brighton TA, Eikelboom JW, Mann K, et al. Low-dose aspirin for preventing recurrent venous thromboembolism. N Engl J Med.2012;367(21):1979-1987.

34. Konstantinides SV, Meyer G, Becattini C, et al. 2019 ESC Guidelines for the diagnosis and management of acute pulmonary embolism developed in collaboration with the European Respiratory Society (ERS).European heart journal. 2019.

35. Hohnloser SH, Pajitnev D, Pogue J, et al. Incidence of stroke in paroxysmal versus sustained atrial fibrillation in patients taking oral anticoagulation or combined antiplatelet therapy: an ACTIVE W Substudy. $J$ Am Coll Cardiol. 2007;50(22):2156-2161.

\section{Figure legends}

Figure 1. Flowchart for selection of the study patients

Figure 2. The cumulative incidence rate of arterial thromboembolic event (A), ischemic stroke (B), extracranial arterial thromboembolic events (C), cardiovascular death (D) and all-cause mortality (E) after propensity score matching comparing patients with venous thromboembolism and those with atrial fibrillation.

Figure 3. The cumulative incidence rate of arterial thromboembolic event (A), ischemic stroke (B), extracranial arterial thromboembolic events (C), cardiovascular death (D) and all-cause mortality (E) after propensity score matching comparing patients with deep vein thrombosis alone and those with atrial fibrillation.

Figure 4. The cumulative incidence rate of arterial thromboembolic event (A), ischemic stroke (B), extracranial arterial thromboembolic events (C), cardiovascular death (D) and all-cause mortality (E) after propensity score matching comparing patients with pulmonary embolism alone and those with atrial fibrillation.

Figure 5: The cumulative incidence rate of arterial thromboembolic event (A), ischemic stroke (B), extracranial arterial thromboembolic events (C), cardiovascular death (D) and all-cause mortality (E) after 
propensity score matching comparing patients with pulmonary embolism alone and those with deep vein thrombosis alone.

Figure 6. The incidence of arterial thromboembolic events and mortality between VTE and AF cohorts. The incidence of ischemic stroke and myocardial infarction were lower in VTE cohort than AF cohort but ECATE, particularly in low extremity, was lower in AF cohort than VTE cohort. In terms of mortality, CV death was lower in VTE cohorts than AF cohort while all-cause mortality and non-CV death were lower in AF cohort than VTE cohort. Abbreviation: AF: atrial fibrillation, CV: cardiovascular; ECATE: extracranial arterial thromboembolic event; VTE: venous thromboembolism.

\section{Hosted file}

table 1.docx available at https://authorea.com/users/336786/articles/509835-differentialpresentations-of-arterial-thromboembolic-events-between-venous-thromboembolism-andatrial-fibrillation-patients
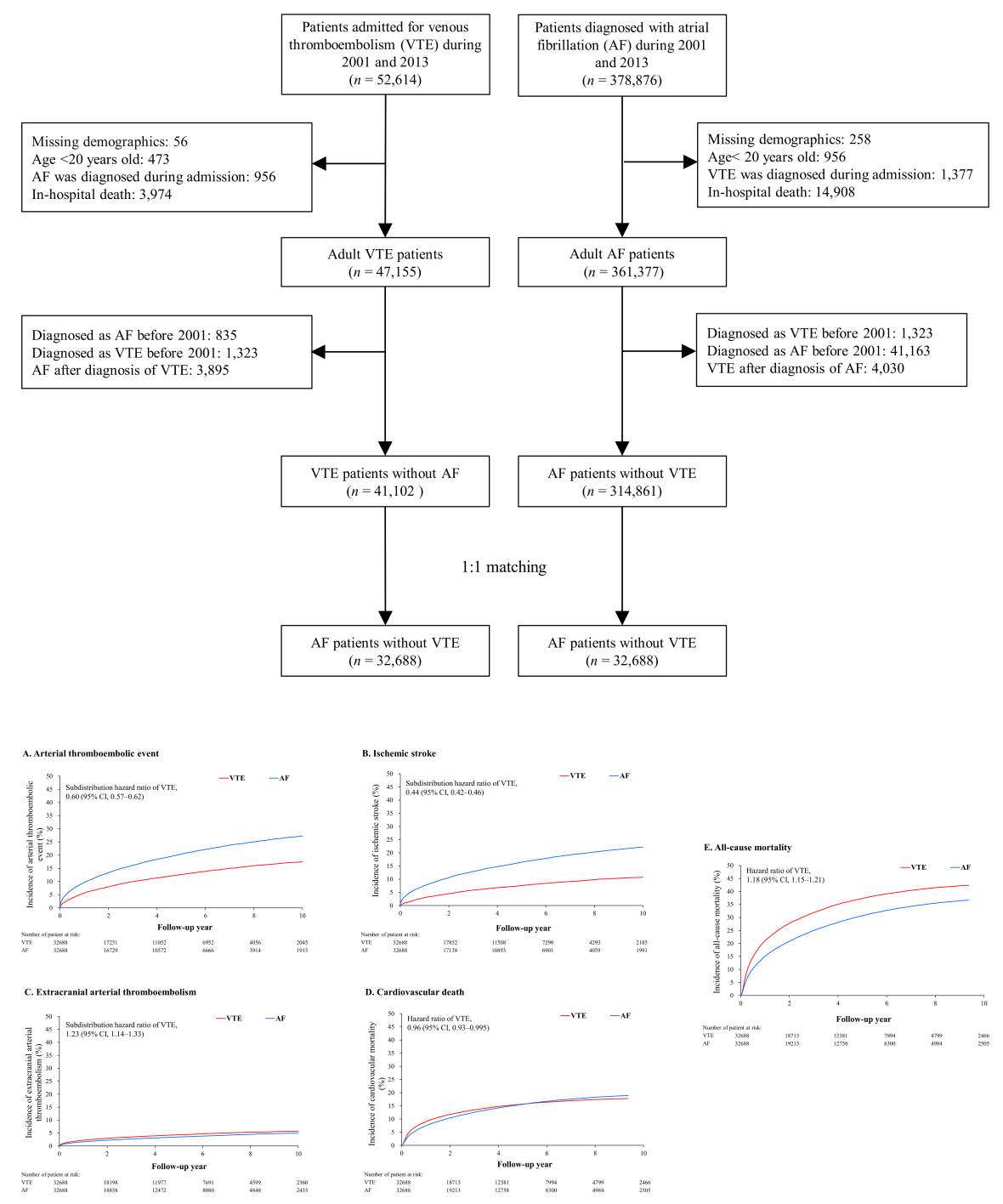

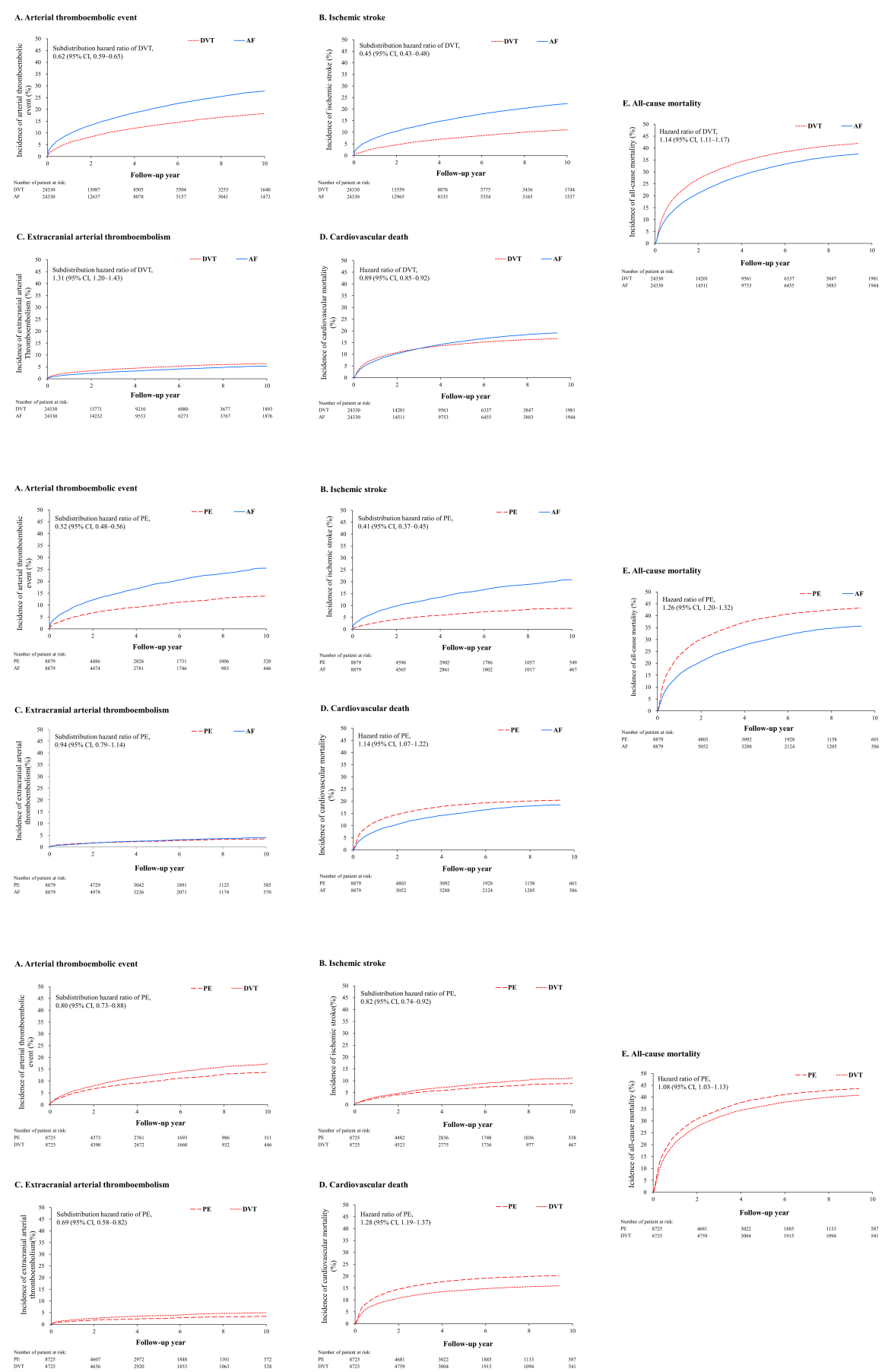


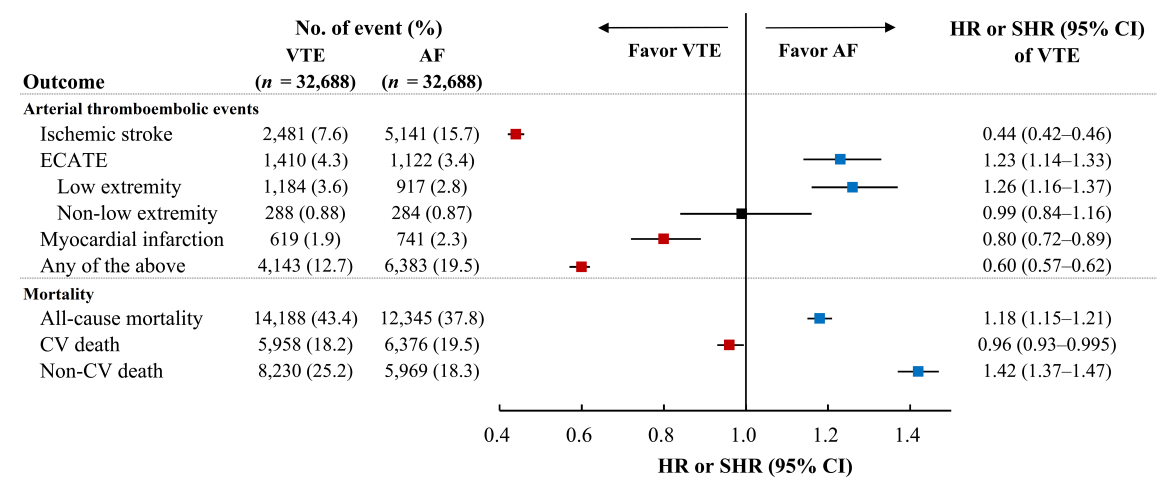

